\title{
Drug Resistance-Associated Mutations in Antiretroviral Treatment-Experienced Patients in Kuwait
}

\author{
Wassim Chehadeh $^{\mathrm{a}}$ Osama Albaksami $^{\mathrm{b}}$ Sonia Elezebeth John ${ }^{\mathrm{a}}$ \\ Widad Al-Nakib ${ }^{\text {a }}$

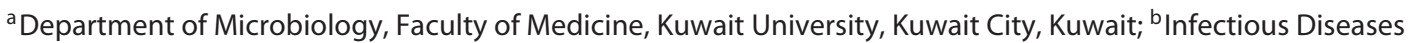 \\ Hospital, Ministry of Health, Kuwait City, Kuwait
}

\section{Significance of the Study}

- The prevalence of resistance-associated mutations in treatment-experienced patients with HIV-1 infection was investigated in Kuwait. Resistance-associated mutations were detected in $26.2 \%$ of the patients. M184V and K103N were the most commonly detected mutations associated with resistance to nucleoside reverse transcriptase inhibitors (NRTI) and non-NRTI (NNRTI), respectively. Monitoring drug resistance in patients on an NNRTI-based regimen is crucial to avoid treatment failure.

\section{Keywords}

HIV-1 · Genotyping · Mutations · Drug resistance ·

Surveillance

\begin{abstract}
Objectives: To investigate the prevalence of nonpolymorphic resistance-associated mutations (RAM) in HIV-1 patients on first-line antiretroviral therapy in Kuwait. Subjects and Methods: Total RNA was isolated from plasma samples of 42 patients who received a first-line nonnucleoside reverse transcriptase inhibitor (NNRTI)-based regimen. HIV-1 protease and reverse transcriptase genetic regions were then amplified by nested reverse transcription-polymerase chain reaction and directly sequenced. The HIV-1 subtype was identified using the Bayesian phylogenetic method, and RAM were identified using the Stanford University genotypic resistance interpretation algorithm. Results: The HIV-1 vi-
\end{abstract}

ral load at sampling ranged from $<20$ to $8.25 \times 10^{4}$ copies/ ml. CRF01_AE, C, and B were the most predominant HIV-1 subtypes. Nonpolymorphic mutations associated with resistance to antiretroviral drugs were detected in 11 (26.2\%) of the 42 patients; 5 (11.9\%) patients had mutations associated with a high-level resistance to nucleoside reverse transcriptase inhibitors (NRTI), 4 (9.5\%) patients had mutations associated with resistance to NNRTI, 1 (2.4\%) patient had mutations associated with resistance to both NRTI and NNRTI, and $1(2.4 \%)$ patient had mutations potentially associated with low-level resistance to both protease inhibitors and NNRTI. All patients with RAM had a detectable plasma HIV-1 RNA level. Conclusion: Our results indicate the development of RAM during an NNRTI-based regimen and highlight the importance of considering other regimens to avoid treatment failure.

(c) 2018 The Author(s)

Published by S. Karger AG, Basel

\begin{tabular}{ll}
\hline KARGER & $\begin{array}{l}\text { @ 2018 The Author(s) } \\
\text { Published by S. Karger AG, Basel }\end{array}$ \\
$\begin{array}{l}\text { Openger } \\
\text { E-Mail karger@karger.coms }\end{array}$ \\
www.karger.com/mpp & $\begin{array}{l}\text { This is an Open Access article licensed under the Creative Commons } \\
\text { Attribution-NonCommercial-4.0 International License (CC BY-NC) } \\
\text { (http://www.karger.com/Services/OpenAccessLicense), applicable to } \\
\text { the online version of the article only. Usage and distribution for com- } \\
\text { mercial purposes requires written permission. }\end{array}$
\end{tabular}

Dr. Wassim Chehadeh

Department of Microbiology, Faculty of Medicine Kuwait University, Street Mubarak Al-Kabir Hospital PO Box 24923, Safat, Kuwait City 13110 (Kuwait) E-Mail wchehadeh@ hsc.edu.kw 


\section{Introduction}

Maintaining a durable HIV suppression is the main goal of antiretroviral therapy (ART) [1]. However, the main cause of therapy failure is the emergence of resistance-associated mutations (RAM) in the HIV pol gene that could lead to accelerated HIV progression and transmission of drug-resistant strains to susceptible individuals $[2,3]$. Drug resistance can either be acquired through drug selection pressure ("acquired resistance" in ARTexperienced patients) or transmitted from person to person ("transmitted resistance" in ART-naive patients) [4]. Kuwait has a low-prevalence of HIV-1 infection because a cumulative total of 252 Kuwaiti HIV cases had been reported until the end of 2013, with 30-50 new cases diagnosed annually [5]. In an attempt to prevent HIV transmission, premarital HIV screening became mandatory in Kuwait in 2009, and all expatriates seeking a residency permit are screened for HIV antibodies. Moreover, antiretroviral treatment is offered free of charge to all infected people. During the study period, the first-line therapeutic regimen in Kuwait was a nonnucleoside reverse transcriptase inhibitor (NNRTI)-based regimen. Protease inhibitor (PI)- and integrase strand transfer inhibitor-based regimens were also available and offered as second- and third-line ART, respectively.

In a pilot study [6] conducted on 28 treatment-naive Kuwaiti patients, a total of 10 different subtypes and recombinant forms were detected, with a predominance of subtypes B, C, and CRF01_AE. Moreover, 2 RAM were detected, one of which was associated with low- to intermediate-level resistance to NNRT. However, drug resistance is more likely to occur wherever HIV therapy is available, and continuing therapy in the context of treatment failure will often lead to the emergence and accumulation of additional resistance mutations $[7,8]$ and, particularly, to drugs with low genetic barriers to resistance [9]. Moreover, drug resistance is likely to occur when individuals do not adhere to anti-HIV drugs [10].

Antiretroviral treatment failure develops in about $20 \%$ of people with HIV receiving first-line ART in lowresource settings [11]. In these cases, a second-line ART was offered to more than half a million people in 2015 [12], and the World Health Organization projects that this number will increase steadily over the next 10 years [13]. In this study, the prevalence of RAM in patients receiving first-line antiretroviral treatment was investigated.

\section{Subjects and Methods}

From June 2011 to March 2016, plasma samples were separated from the blood of 42 patients under more than 6 months of a firstline NNRTI-based regimen. Their age ranged from 4 months to 65 years, with a median age of 33 years. The treatment consisted of efavirenz or nevirapine with a combination of 2 NRTI, i.e., lamivudine or emtricitabine + tenofovir or abacavir. The exclusion criterion was evidence of nonadherence to ART. Informed consent was obtained from each patient. The Ethics Committee, Faculty of Medicine, Kuwait University, and the Standing Committee for Coordination of Health and Medical Research, Ministry of Health, approved this study.

\section{HIV RNA Concentrations}

The HIV-1 RNA concentrations in the plasma samples of recently diagnosed HIV-1 patients were determined by real-time PCR using the COBAS AmpliPrep/COBAS TaqMan HIV-1 test v2.0 (Roche Diagnostic Systems, Branchburg, NJ, USA) as per the manufacturer's instructions. The limit of quantification, as defined by the manufacturer, was 20 copies $/ \mathrm{ml}$.

\section{HIV Subtype Classification}

The automated MagNa Pure LC 2.0 system (Roche Diagnostic Systems) was used to isolate the viral RNA from the clinical samples. In-house amplification of a 1,300-bp fragment of the pol gene by nested reverse transcription-polymerase chain reaction was carried out as described previously [14]. The PCR products were purified using Wizard SV GEL and a PCR Clean-Up System Kit (Promega Corporation, Madison, WI, USA), and the nucleotide sequences of both DNA strands were then determined by direct double-strand DNA cycle sequencing using an $\mathrm{ABI}$ PRISM ${ }^{\circledR}$ BigDye $^{\circledR}$ Terminator Cycle Sequencing v3.1 Kit (Applied Biosystems, Foster City, CA, USA). Post sequencing PCR purification was performed to remove unbound fluorescent dye deoxy terminators using a BigDye XTerminator ${ }^{\mathrm{TM}}$ Purification Kit (Applied Biosystems). The samples were then denatured for 2 min at $95^{\circ} \mathrm{C}$, immediately chilled on ice, and loaded on an ABI 3500 Genetic Analyzer (Applied Biosystems). DNA sequences were then subjected to electrophoresis on a 50-cm 8-capillary array using POP7TM polymer (Applied Biosystems) as a separation medium and analyzed using 3500 Series Data Collection Software version 3 and Sequencing Analysis Software version 6 (Applied Biosystems). The obtained nucleotide sequences consisting of the pol gene were searched against the NCBI GenBank database using Basic Local Alignment Search Tool (BLAST) and then aligned with all of the HIV reference sequences available in the HIV sequence database [15] using the ClustalW method in Molecular Evolutionary Genetics Analysis (MEGA) software version 4.02 [16]. Phylogenetic trees were reconstructed with the Bayesian Markov Chain Monte Carlo approach implemented in the BEAST v.1.7.4 program [17]. The Bayesian Skyline plot was run under a relaxed uncorrelated log normal molecular clock using the best model of nucleotide substitution (HKY) as the closest related to that obtained in MODELTEST. Convergence of the parameters during the Markov Chain Monte Carlo was inspected with Tracer v.1.5 [17], with uncertainties addressed as the $95 \%$ highest probability density intervals. Ten million chains were sufficient to achieve convergence of all parameters, with an effective sampling size (>200). The trees were sampled at each 1,000 steps, resulting in a final file of 10,000 trees. 
These trees were summarized in a maximum a posteriori tree using the TreeAnotator v.1.7.4 program (part of the BEAST package) and visualized with the FigTree v.1.4.0 program [18].

HIV-1 sequences resulting from this study were uploaded to the GenBank database (accession No. KX155586 to KX155647). The recombinant HIV-1 forms were analyzed by performing a bootscan analysis with a sliding window of $200 \mathrm{bp}$, incremental steps of 20 bases, and the Kimura 2-parameter model using Simplot 3.5.1 software (Johns Hopkins University, Baltimore, MD, USA). The boostscan analysis was performed first with sequences only from pure subtypes and then with all of sequences including recombinant forms available in the HIV sequence database. All subtypes and circulating recombinant forms (CRF) were confirmed using the REGA HIV subtyping tool [19].

\section{Assessment of Drug Resistance}

HIV-1 drug resistance was determined by sequencing the reverse transcriptase and protease regions on the HIV-1 pol gene. The prevalence of drug resistance was estimated using the Stanford University genotypic resistance interpretation algorithm [20].

\section{Statistical Analysis}

The two-tailed Mann-Whitney $U$ test was used to assess the difference in HIV-1 viral loads between 2 groups. Statistical analysis was performed using the IBM Statistical Package for the Social Sciences (SPSS) Statistics for Windows, version 23 (IBM Corp., Armonk, NY, USA).

\section{Results}

\section{Baseline Characteristics of the Patients}

The baseline characteristics of treatment-experienced patients are given in Table 1. Most patients were male Kuwaitis. The median viral load at sampling in treatment-experienced patients was $3.76 \times 10^{2} \mathrm{RNA}$ copies $/ \mathrm{ml}$ (range: $<20$ to $8.25 \times 10^{4}$ )

\section{Subtype Distribution}

Based on the phylogenetic analysis of the partial pol gene (Fig. 1; Table 1), the decreasing order of the prevalences of the subtypes was: CRF01_AE, 33\%; subtype C, $31 \%$; subtype $B, 14 \%$; subtype G, $5 \%$; and subtype A, $2 \%$. Six patients had a recombinant form of HIV-1 different from CRF01_AE; among them, 4 had CRF02_AG, 1 had CRF35_AD, and 1 had the CRF63_02A1 subtype (Fig. 1).

\section{Drug Resistance Profile}

The RAM in HIV-1 patients on ART are listed in Table 2. Of the 42 patients, $11(26.2 \%)$ had nonpolymorphic mutations associated with resistance to antiretroviral drugs. Among patients with mutations associated with resistance to NRTI, 5 (11.9\%) had the M184V mutation associated with high-level resistance to lamivudine (3TC)
Table 1. Baseline characteristics of treatment-experienced HIV patients

$\begin{array}{lc}\text { Gender } & 29(69) \\ \text { Male } & 13(31) \\ \text { Female } & 33(4 \text { months to } 65 \text { years) } \\ \text { Age, years } & 33(79) \\ \text { Nationality } & 9(21) \\ \text { Kuwaiti } & \\ \text { Non-Kuwaiti } & 19(45) \\ \text { Viral load } & 23(55) \\ \leq 10^{4} \text { copies/ml } & 0(0) \\ 10^{4}-10^{5} \text { copies/ml } & \\ \geq 10^{5} \text { copies/ml } & 1(2) \\ \text { HIV-1 subtype } & 6(14) \\ \text { A } & 13(31) \\ \text { B } & 2(5) \\ \text { C } & 14(33) \\ \text { G } & 4(10) \\ \text { CRF01_AE } & 1(2) \\ \text { CRF02_AG } & 1(2) \\ \text { CRF35_AD } & \end{array}$

Values are presented as numbers (\%) or medians (range).

and emtricitabine (FTC) and low-level resistance to didanosine (ddI) and abacavir (ABC). Among patients with mutations associated with resistance to NNRTI, 2 patients had the K103N mutation associated with high-level resistance to nevirapine (NVP) and efavirenz (EFV); 1 patient had K103N and Y181C mutations associated with high-level resistance to NVP and EFV and intermediate resistance to etravirine (ETR) and rilpivirine (RPV); 1 patient had A98G and K101E mutations associated with high-level resistance to NVP, intermediate resistance to RPV, and low-level resistance to EFV and ETR; 1 patient had the V179T mutation potentially associated with lowlevel resistance to all available NNRTI. One patient with an HIV-1 subtype B infection had multiple RAM (M184V, K219Q, V108I, E138K, F227L, and M230L) known to confer high-level resistance to 3TC, FTC, EFV, NVP, and $\mathrm{RPV}$, intermediate resistance to ETR, and low-level resistance to $\mathrm{ABC}$ and ddI.

The mutation profiles were consistent with the treatment status, with K103N and Y181C mutations identified in patients treated with EFV or NVP, and M184V mutations found in patients treated with 3TC. However, in 1 patient under an NNRTI-based regimen with no history of previous exposure to PI, 2 RAMs were detected, i.e., L10F potentially conferring low-level resistance to the PI fosamprenavir/ritonavir (FPV/r), indinavir/r (IDV/r), 
Fig. 1. Phylogenetic analysis of a partial fragment of the HIV pol gene. Bayesian phylogenetic analysis of a $~ 1,300$-bp fragment of the HIV-1 pol gene. The values of posterior probability are depicted at the nodes of the trees. Each reference HIV-1 sequence is labeled with its corresponding subtype. Each HIV-1 sequence from this study is labeled with a star. Scale is in substitutions/site. For clarity of the figure, reference HIV-1 sequences from recombinant forms not relevant to samples from this study were removed.

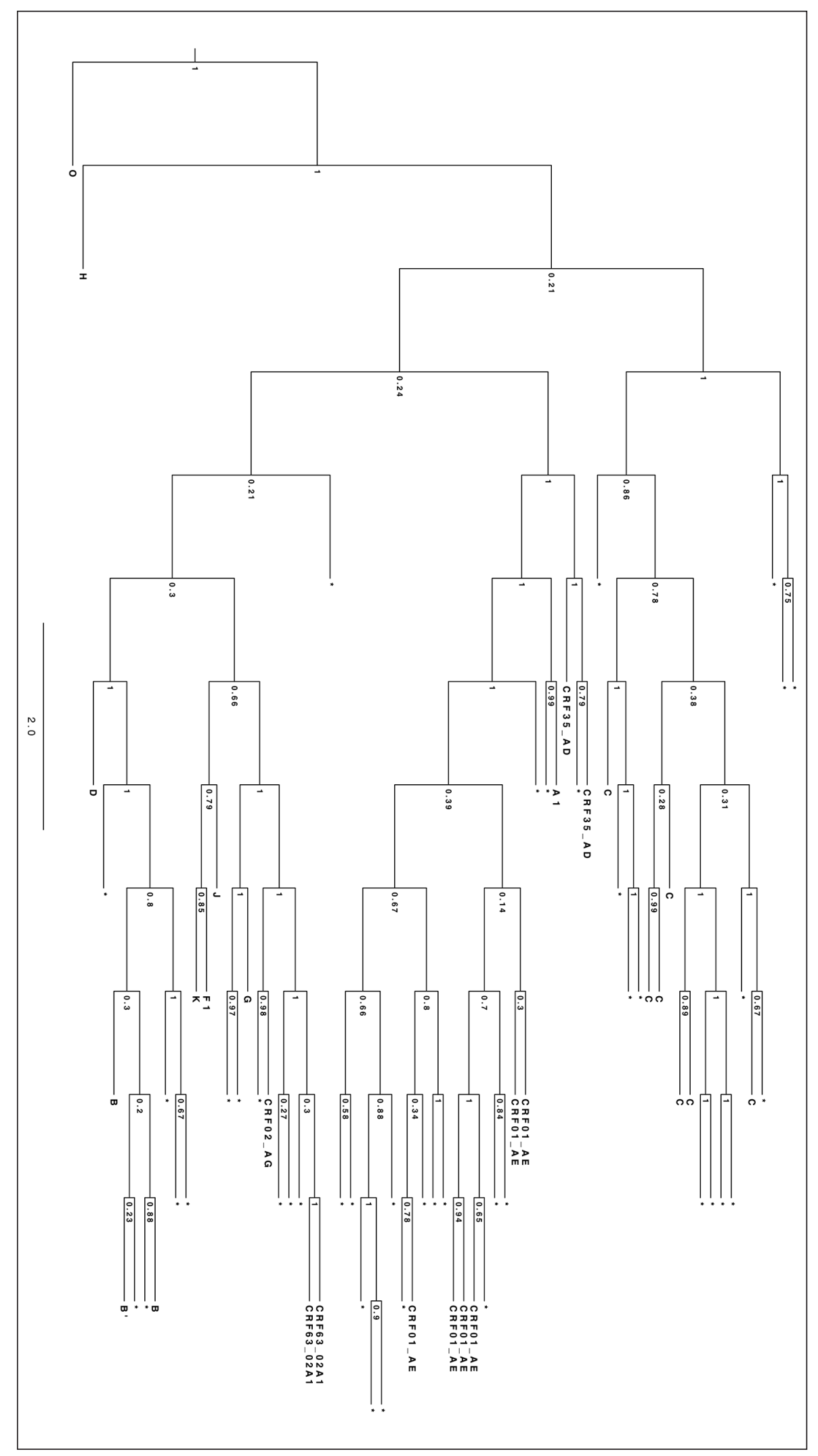

Med Princ Pract 2018;27:152-157 
Table 2. RAM in HIV-1 patients on ART

\begin{tabular}{|c|c|c|c|c|}
\hline K70T & NRTI & Potential low & $\mathrm{C}$ & $1(2.4)$ \\
\hline K103N & NNRTI & High & A, CRF01_AE & $2(4.8)$ \\
\hline M184V & NRTI & High & CRF01_AE & $2(4.8)$ \\
\hline $\mathrm{L} 10 \mathrm{~F}+\mathrm{V} 179 \mathrm{~T}$ & $\mathrm{PI}+\mathrm{NNRTI}$ & Potential low & CRF02_AG & $1(2.4)$ \\
\hline $\mathrm{K} 70 \mathrm{E}+\mathrm{M} 184 \mathrm{~V}$ & NRTI & High & CRF01_AE & $1(2.4)$ \\
\hline $\mathrm{K} 103 \mathrm{~N}+\mathrm{Y} 181 \mathrm{C}$ & NNRTI & High & CRF01_AE & $1(2.4)$ \\
\hline $\mathrm{M} 184 \mathrm{~V}+\mathrm{K} 219 \mathrm{Q}+\mathrm{V} 108 \mathrm{I}+\mathrm{E} 138 \mathrm{~K}+\mathrm{F} 227 \mathrm{~L}+\mathrm{M} 230 \mathrm{~L}$ & NRTI+NNRTI & High & $\mathrm{B}$ & $1(2.4)$ \\
\hline Total & & & & $11(26.2)$ \\
\hline
\end{tabular}

RAM, resistance-associated mutations; ART, antiretroviral therapy; NRTI, nucleoside reverse transcriptase inhibitor; NNRTI, nonnucleoside reverse transcriptase inhibitor; PI, protease inhibitor.

and nelfinavir (NFV), and V179T potentially associated with low-level resistance to all available NNRTI. All patients with high-level resistance to ART $(n=9 ; 21.4 \%)$ had detectable plasma HIV-1 RNA levels, with median viral load of $4.69 \times 10^{3} \mathrm{RNA}$ copies/ml (range: $2.85 \times 10^{2}$ to $6.49 \times 10^{4}$ RNA copies $\left./ \mathrm{ml}\right)$.

\section{Discussion}

In this study, the HIV-1 subtypes CRF01_AE, C, and $B$ were found to be the most prevalent subtypes detected in Kuwait, consistent with previous observations [6] and the findings reported in the Gulf region about the circulation of pure subtypes $\mathrm{A}, \mathrm{B}$, and $\mathrm{C}$ and of recombinant forms like CRF35_AD [21, 22]. Of interest, around 10\% of the patients had the HIV-1 subtype CRF02_AG that is prevalent in West Africa and Russia with a global prevalence of $\sim 5 \%$ [23]. Moreover, 1 Kuwaiti patient had the subtype CRF63_02A1 that was described in Russia [24].

A total of $26.2 \%$ of patients had mutations known to be associated with resistance to antiretroviral drugs. About half of them had more than one primary mutation which may result in greater reductions in susceptibility and virologic response than does a single mutation. M184V was the predominant mutation associated with high-level resistance to 3TC and FTC and low-level resistance to ddI and $\mathrm{ABC}$. However, M184V is not a contraindication to continued treatment with $3 \mathrm{TC}$ or
FTC because it increases the susceptibility to zidovudine (AZT), tenofovir (TDF), and stavudine ( $\mathrm{d} 4 \mathrm{~T})$ and is associated with clinically significant reductions in HIV-1 replication [25]. K103N was the second most common detected mutation, and it is known to confer high-level resistance to the first-generation NNRTI EFV and NVP, as described above. The presence of $\mathrm{K} 103 \mathrm{~N}$ alone does not affect the response to the second-generation NNRTI ETR and RPV; however, the accumulation of several mutations will compromise their efficacy [26]. Y181C, detected in 1 treatment-experienced patient, is a nonpolymorphic mutation selected in patients receiving NVP, ETR, and RPV. It reduces the susceptibility to NVP, ETR, RPV, and EFV by $>50$-fold, 5-fold, 3-fold, and 2-fold, respectively. Although Y181C itself reduces EFV susceptibility by only 2 -fold, it is associated with a reduced response to an EFV-containing regimen because viruses with this mutation often harbor additional minority variant NNRTI-resistance mutations [27]. In addition to the Y181C mutation, E138K and K101E, detected in 2 different treatment-experienced patients, are associated with a 2- to 3-fold reduced susceptibility to RPV. They occur commonly in patients receiving rilpivirine. E138K and to a lesser extent K101E usually occur in combination with the NRTI resistance mutation M184I, which alone does not reduce rilpivirine susceptibility. When M184I is combined with E138K or K101E, rilpivirine susceptibility is reduced about 7 -fold and 4.5fold, respectively [28]. 
In patients receiving a first-line treatment regimen, the prevalence of mutations associated with resistance to NRTI, NNRTI, and PI was $14.3,14.3$, and $2.4 \%$, respectively. In Saudi Arabia, $41 \%$ of treatment-experienced patients were reported to have NRTI resistance mutations, $16 \%$ had NNRTI resistance mutations, and $13 \%$ had PI resistance mutations [29]. In Iran, the prevalence of NRTI, NNRTI, and PI resistance mutations was reported to be $\sim 50,29$, and $6.5 \%$, respectively [30]. Data from neighboring countries are currently too scarce to evidence sizable discrepancies between results.

\section{Conclusions}

The prevalence of RAM was $26.2 \%$ in patients on a first-line treatment regimen, and approximately half of the patients had more than 1 RAM. The most commonly detected mutations were M184V associated with resistance to NRTI and K103N associated with resistance to first-generation NNRTI. However, primary mutations associated with high-level resistance to PI were not detected. These findings indicate the necessity for continuous surveillance of drug resistance in patients to reduce the likelihood of treatment regimen failure.

\section{References}

1 Walensky RP, Paltiel AD, Losina E, et al: The survival benefits of AIDS treatment in the United States. J Infect Dis 2006;194:11-19.

2 Little SJ, Holte S, Routy JP, et al: Antiretroviral-drug resistance among patients recently infected with HIV. N Engl J Med 2002;347: 385-394.

3 Richman DD, Margolis DM, Delaney M, et al: The challenge of finding a cure for HIV infection. Science 2009;323:1304-1307.

4 Tang MW, Shafer RW: HIV-1 antiretroviral resistance: scientific principles and clinical applications. Drugs 2012;72:e1-e25.

5 UNAIDS: Global AIDS Response Progress Report 2014: Country Progress Report - State of Kuwait. http://www.unaids.org/en/regionscountries/countries/kuwait/ (accessed October 22, 2014).

6 Chehadeh W, Albaksami O, Altawalah H, et al: Phylogenetic analysis of HIV-1 subtypes and drug resistance profile among treatmentnaïve people in Kuwait. J Med Virol 2015;87: 1521-1526.

7 Preston BD, Dougherty JP: Mechanisms of retroviral mutation. Trends Microbiol 1996; 4:16-21.

8 Roberts JD, Bebenek K, Kunkel TA: The accuracy of reverse transcriptase from HIV-1. Science 1998;242:1171-1173.

9 Hirsch MS, Gunthard HF, Schapiro JM, et al: Antiretroviral drug resistance testing in adult HIV-1 infection: 2008 recommendations of an International AIDS Society-USA panel. Clin Infect Dis 2008;47:266-285.

10 Emamzadeh-Fard S, Fard SE, SeyedAlinaghi $S$, et al: Adherence to anti-retroviral therapy and its determinants in HIV/AIDS patients: a review. Infect Disord Drug Targets 2012;12: 346-356.
11 Zhang F, Dou Z, Ma Y, et al: Five year outcomes of the China National Free Antiretroviral Treatment Program. Ann Intern Med 2009;151:241-251.

12 Haas AD, Keiser O: Raltegravir in second-line ART in resource-limited settings. Lancet HIV 2016;3:e240-e241.

13 World Health Organization: WHO HIV drug resistance report 2012. http://www.who.int/ hiv/pub/drugresistance/report2012/en/ (accessed October 14, 2014).

14 World Health Organization: WHO manual for HIV drug resistance testing using dried blood spot specimens. 2012. http://www.who. int/hiv/pub/drugresistance/en/ (accessed October 14, 2014)

15 HIV sequence database. http://www.hiv.lanl. gov/ (accessed May 16, 2013).

16 Tamura K, Dudley J, Nei M, et al: Molecular Evolutionary Genetics Analysis (MEGA) software version 4.0. Mol Biol Evol 2007;24: 1596-1599.

17 Drummond AJ, Rambaut A: BEAST: Bayesian evolutionary analysis by sampling trees. BMC Evol Biol 2007;7:214.

18 FigTree software. http://beast.community/ figtree (accessed October 14, 2014).

19 REGA HIV subtyping tool. http://http:// www.bioafrica.net/rega-genotype/html/subtypinghiv.html (accessed October 14, 2014).

20 Liu TF, Shafer RW: Web resources for HIV type 1 genotypic-resistance test interpretation. Clin Infect Dis 2006;42:1608-1618.

21 Alzahrani AJ: Analysis of HIV subtypes and the phylogenetic tree in HIV-positive samples from Saudi Arabia. Saudi Med J 2008;29: 1394-1396.

22 Baesi K, Moallemi S, Farrokhi M, et al: Subtype classification of Iranian HIV-1 sequences registered in the HIV databases, 20062013. PLoS One 2014;9:e105098.
23 Taylor BS, Sobieszczyk ME, McCutchan FE, et al: The challenge of HIV-1 subtype diversity. N Engl J Med 2008;358:1590-1602.

24 Shcherbakova NS, Shalamova LA, Delgado E, et al: Molecular epidemiology, phylogeny, and phylodynamics of CRF63_02A1, a recently originated HIV-1 circulating recombinant form spreading in Siberia. AIDS Res Hum Retroviruses 2014;30:912-919.

25 Kuritzkes DR, Quinn JB, Benoit SL, et al: Drug resistance and virologic response in NUCA 3001, a randomized trial of lamivudine (3TC) versus zidovudine (ZDV) versus ZDV plus 3TC in previously untreated patients. AIDS 1996;10:975-981.

26 Tudor-Williams G, Cahn P, Chokephaibulkit $\mathrm{K}$, et al: Etravirine in treatment-experienced, HIV-1-infected children and adolescents: 48week safety, efficacy and resistance analysis of the phase II PIANO study. HIV Med 2014;15: 513-524.

27 Shafer RW, Dupnik K, Winters MA, et al: A Guide to HIV-1 Reverse Transcriptase and Protease Sequencing for Drug Resistance Studies. HIV Seq Compend 2001;2001:1-51.

28 Kulkarni R, Babaoglu K, Lansdon EB, et al: The HIV-1 reverse transcriptase M184I mutation enhances the E138K-associated resistance to rilpivirine and decreases viral fitness. J Acquir Immune Defic Syndr 2012;59:47-54.

29 Jamjoom GA, Azhar EI, Madani TA, et al: Genotype and antiretroviral drug resistance of human immunodeficiency virus-1 in Saudi Arabia. Saudi Med J 2010;31:987-992.

30 Baesi K, Ravanshad M, Ghanbarisafari M, et al: Antiretroviral drug resistance among antiretroviral-naïve and treatment experienced patients infected with HIV in Iran. J Med Virol 2014;86:1093-1098. 